\title{
Estrategias para mejorar la convivencia entre los educandos de la ERM la Pacha ${ }^{1}$
}

\section{Strategies to improve coexistence among the students of the ERM la Pacha}

DOI: http://dx.doi.org/10.17981/cultedusoc.9.3.2018.35

\author{
Iris Avendaño2; \\ Abel Ospino; Alicia González; Audis Rangel; \\ Denis Terraza; Erasmo Terraza; Eunice Carrascal y Eva Vega ${ }^{3}$ \\ ERM la Pacha (Colombia) \\ iravi10@hotmail.com
}

Para citar este artículo:

Avendaño, I., Ospino, A., González, A., Rangel, A.,Terraza, D., Terraza, E., Carrascal, E. y Vega, E. (2018). Estrategias para mejorar la convivencia entre los educandos de la ERM la Pacha. Cultura. Educación y Sociedad 9(3), 309-316. DOI: http://dx.doi.org/10.17981/ cultedusoc.9.3.2018.35

\section{Resumen}

Los valores resultan el principio que conlleva a disfrutar de una buena y sana calidad de vida de acordes con el amor, respeto y tolerancia y La práctica cotidiana de los valores, la experiencia en familia, el uso en ejercicio son la mejor manera de mantenerlos presente. Por tal motivo el objetivo de la presente investigación es rescatar los valores que permitan una sana convivencia y el sentido de pertenencia en la familia, en la escuela y en la comunidad en general. El estudio se sustenta desde una mirada cualitativa, de alcance descriptivo, se aplicó una entrevista estructurada a los estudiantes de la ERM de la Pacha. Como resultados se pudo evidenciar que existe un trabajo mancomunado por la comunidad educativa para crear ambientes armoniosos en el alumnado, sin embargo, se debe seguir fortaleciendo el corporativismo y la participación de la familia como eje central de la enseñanza.

Palabras clave: Valores; familia; convivencia; calidad de vida.

\section{Abstract}

Values are the principle that leads to enjoy a good and healthy quality of life in accordance with love, respect and tolerance and The daily practice of values, family experience, use in exercise are the best way to keep them present. For this reason, the objective of the present investigation is to rescue the values that allow a healthy coexistence and the sense of belonging in the family, in the school and in the community in general. The study is supported from a qualitative perspective, descriptive in scope, a structured interview was applied to the students of the ERM of the Pacha. As results, it was possible to demonstrate that there is a joint effort by the educational community to create harmonious environments in the students, however, we must continue strengthening the corporatism and the participation of the family as the central axis of the teaching.

Keywords: Values; family; coexistence; quality of life.

\footnotetext{
1 Este artículo ha sido derivado del Programa de Fortalecimiento de la Cultura Ciudadana y Democrática CT+I a través de la IEP apoyada en TIC en el Departamento de Magdalena: CICLON.

2 Líder del grupo de investigación "Buscando Amor".

3 Docentes pertenecientes al grupo de investigación "Buscando Amor" de la ERM la Pacha. Proyecto Ciclón, Magdalena, Estrategia $\mathrm{N}^{\circ}$ 2. Universidad de la Costa CUC.
}

The author; licensee Universidad de la Costa - CUC 


\section{Introducción}

El tema del ser humano y sus valores, continua vigente, ha estado presente constantemente en la historia de la humanidad. Se escoge ser honestos o no, hacer el bien o burlarse del vecino, agradecerle a la naturaleza sus frutos o pisotearla o maltratarla. Se podría ser inconsecuentes con los maestros o ser más justos y prudentes, ser irrespetuosos e intolerantes o tomar la decisión más adecuada y prudente. Todas estas formas tan diferentes de expresar lo que se siente llevan a una implícita actitud que se cultiva con el paso del tiempo.

En esto radica precisamente la esencia de desarrollar este proyecto, en saber elegir la opción más adecuada, humana y tolerante. Esto es lo que conlleva a que los seres humanos puedan convivir de una manera sana con su entorno.

La curiosidad es el deseo primordial que mueve la infancia del ser humano. Si se forman a los estudiantes en la práctica de los buenos valores morales, se logrará transformar toda la civilización transmitiéndole enseñanzas a través de la reflexión y no de reglas ni mucho menos del castigo.

El Desarrollo de proyectos como estos contribuye a fortalecer la capacidad de amar, para sentir alegría, para ver en forma simple la grandeza de la naturaleza, para cambiar y hacer de su propia vida, para hacer realidad sus metas y los sueños que solo le producen felicidad.

La práctica cotidiana de los valores, la experiencia en familia, el uso en ejercicio son la mejor manera de mantenerlos presente. Los valores no son otra cosa que principios para disfrutar una buena y sana calidad de vida acordes con el amor, el respeto y la tolerancia. Para hacer realidad esta visión nosotros como maestros o padres debemos contar con principios prácticos que podamos enseñarles, cuanto más aprendamos a vivir en armonía y creativamente mayor será la probabilidad de experimentar el éxito durante toda la vida, ya que el éxito depende de lo que somos no de lo que hacemos (Avendaño, Cortés y Guerrero, 2015).

El objetivo de la presente investigación fue rescatar los valores que permitan una sana convivencia y el sentido de pertenencia en la familia, en la escuela y en la comunidad.

Para para dar respuesta al objeto de estudio se creó el proyecto Con-Vive y Sé Feliz, se convirtió en un plan de acción que buscó la mejora en la convivencia de todo el centro y mejorar las relaciones interpersonales entre alumnos, docentes, no docentes y familias. Cuya problemática planteada fue una serie de conflictos y problemas de convivencia, sobre todo en el último ciclo de primaria. Este se fundamentó en que la enseñanza de la convivencia debe estar basada en colocar en práctica los valores, lo cual ayuda a desarrollar aquellas destrezas que integran la "inteligencia moral y emocional". "El conocimiento de uno mismo, la empatía, la comprensión crítica, el juicio moral, la autonomía, la solidaridad, etc." (Acosta \& Mesas, 2008).

\section{Escuela y valores}

\footnotetext{
"El reto del siglo XXI sigue siendo el de definir los derechos humanos en términos capaces de sostener el principio de la igualdad a partir del reconocimiento de la diversidad" (Nash, 1993. p.32.).
} 
Muchas veces el entorno es terriblemente hostil para la formación de la infancia debido a falta de estímulos, a un desierto cultural, como consecuencia de la dispersión del territorio.

La educación termina en la escuela, por lo que tiene que llegar su cultura hasta la cultura de la ciudad" Escuela y Entorno, (Carbonell, 2001). La escuela trata de mantener la cultura escolar excluida de la mala cultura externa, siendo los medios de comunicación los que más abonan a la cultura del niño. El contexto educa más que el texto. Se debe tener en cuenta que el entorno es uno de los factores influyentes y no siempre es el más adecuado, puesto que este no distingue la infancia, adolescencia, etc.; por ello se está propenso a cambiar la conducta en este diverso choque de culturas, los cuales no tienen un fin en común.

La educación en la escuela, debe fortalecer la dimensión humana, desde una práctica pedagógica donde se desarrollen no solo los contenidos propios del área de conocimiento de cada disciplina, sino contenidos actitudinales es decir, aquellos contenidos relacionados con el saber ser y convivir, respetando las particularidades del sujeto individual que hace parte de un colectivo o sociedad generando, de esta manera, ambientes favorables para su aprendizaje y desarrollo integral (Martínez, Berthel \& Diaz, 2017). Al respecto, De la Concepción (2015), destacan que en el sistema social los ambientes de aprendizaje pueden ser fortalecidos desde mecanismos conscientes de participación como base para el desarrollo de la ciudadanía.

"Pensar en una educación de tolerancia y pluralismo, significa que la escuela debe re contextualizar los elementos universales en particularidades específica de cada cultura, visible a través de cada centro, de cada docente, de cada alumno, contexto que nos permitirá hablar de la nueva comunidad educativa" (Mejía, s.f) ; una comunidad donde converjan el respeto de los valores, de las diferencias y dignidad humana. "De tal manera que la educación resuelve este conflicto, se estarán colocando las bases para la construcción de una democracia profundamente universal, pero levantada desde lo local y desde los regional." (Mejía, s.f).

En el caso de Colombia, coinciden Araque y Suarez (2017), una acción educativa fundada en la responsabilidad ética de las universidades en Colombia puede promover una sociedad del saber, que oriente al conocimiento al desarrollo de estrategias de inclusión social, comprensión común y equilibrio social

\section{Metodología}

Este estudio se llevó a cabo desde un enfoque Cualitativo, tal como lo afirma (Martínez, 2006) este tipo de investigación estudia la realidad en su contexto natural, identificando la naturaleza de la realidad asociada con su comportamiento y sus manifestaciones, además, en su dimensión dinámica busca cumplir las dos tareas básicas de toda investigación: recoger datos y categorizarlos e interpretarlos.

\section{Escenarios y actores}

El escenario donde se desarrolló la investigación es la sede ERM La Pacha del Municipio de San Sebastián Magdalena, con la participación de (30) estudiantes de básica secundaria. 


\section{Técnicas e instrumentos de recolección de la información}

Dado el tipo de investigación cualitativa y las características del escenario de investigación se decidió utilizar como técnicas de recolección: la entrevista estructurada y la observación directa, la cual fue registrada en un diario de campo.

\section{Procedimiento}

La ejecución de la presente investigación está enmarcada en los siguientes momentos:

Momento (1): Elección del fenómeno a estudiar.

Momento (2): Hacer el sondeo diagnóstico de los objetos de interés.

Momento (3): Definición de las categorías de estudio: Convivencia escolar, bulliyng, manual de convivencia, política educativa.

Momento (4): Definición de los instrumentos de recolección de la información.

Momento (5): Aplicación de la entrevista estructurada y los registros de observación. El instrumento fue valorado por jueces a expertos para la validez de contenido, luego fue aplicado a los estudiantes de la IED Externado de San Sebastián. Posterior a ello se realizó un análisis de contenido el cual incluyó una serie de actividades interpretativas, enfocadas a la interpretación de las ideas que asociadas a la voz de los informantes permitió la comprensión del objeto de investigación.

Momento (6): Sistematización de la información a la luz de los datos recolectados y articulación con la teoría.

Momento (7): Generación de resultados.

Momento (8): Socialización de los hallazgos encontrados.

\section{Resultados}

A continuación, se presentan lo hallazgos del estudio, derivados de la entrevista estructurada y el diario de campo, asi mismo se permitió la articulación teórica de acuerdo a los postulados de los autores de base.

\begin{tabular}{|c|c|c|c|}
\hline $\begin{array}{l}\text { Categoría } \\
\text { de estudio }\end{array}$ & $\begin{array}{l}\text { Pregunta } \\
\text { orientadora }\end{array}$ & $\begin{array}{l}\text { Discurso de } \\
\text { los actores }\end{array}$ & $\begin{array}{c}\text { Articulación y } \\
\text { sistematización teórica }\end{array}$ \\
\hline $\begin{array}{l}\text { Convivencia } \\
\text { escolar }\end{array}$ & $\begin{array}{l}\text { ¿Cuáles } \\
\text { son las } \\
\text { situaciones de } \\
\text { convivencias } \\
\text { que se } \\
\text { evidencian en } \\
\text { la ERM La } \\
\text { Pacha? }\end{array}$ & $\begin{array}{l}\text { "Se trata de acabar con } \\
\text { la agresividad entre los } \\
\text { demás para vivir en paz"; } \\
\text { "Convivir con los compañeros, } \\
\text { ayudarlos a jugar hacer } \\
\text { tareas"; "Hay peleas entre } \\
\text { todos los estudiantes"; "Jugar } \\
\text { con los compañeros"; "Ser } \\
\text { amable con los compañeros, } \\
\text { profesores y padres"; }\end{array}$ & $\begin{array}{l}\text { De acuerdo a los hallazgos } \\
\text { encontrados en la entrevista } \\
\text { aplicada, se deduce que los } \\
\text { estudiantes reconocen que se } \\
\text { presentan situaciones que afectan } \\
\text { la convivencia entre los educandos } \\
\text { de la ERM La pacha. } \\
\text { Frente a esto el autor (Paz, 2010) } \\
\text { explica }\end{array}$ \\
\hline
\end{tabular}




\begin{tabular}{|c|c|c|c|}
\hline $\begin{array}{l}\text { Categoría } \\
\text { de estudio }\end{array}$ & $\begin{array}{c}\text { Pregunta } \\
\text { orientadora }\end{array}$ & $\begin{array}{l}\text { Discurso de } \\
\text { los actores }\end{array}$ & $\begin{array}{c}\text { Articulación y } \\
\text { sistematización teórica }\end{array}$ \\
\hline $\begin{array}{l}\text { Convivencia } \\
\text { escolar }\end{array}$ & & $\begin{array}{l}\text { "Groserias, agresividad, falta } \\
\text { de respeto"; "Agresividad, } \\
\text { los niños no saben dialogar } \\
\text { en paz por eso el grupo } \\
\text { Buscando amor quiere } \\
\text { orientarlos para que no sean } \\
\text { agresivos"; "No hay respeto, } \\
\text { no son amables, pelean unos } \\
\text { a otros"; "Humillaciones"; } \\
\text { "Intolerancia, falta de } \\
\text { comunicación entre los niños } \\
\text { de la ERM"; "Peleas entre } \\
\text { los niños"; "La convivencia } \\
\text { que se evidencia en la ERM } \\
\text { La Pacha es respetar a los } \\
\text { semejantes, querernos unos a } \\
\text { otros y tolerar a los demás". }\end{array}$ & $\begin{array}{l}\text { "Desde la psicología existen } \\
\text { muchas teorías sobre las causas } \\
\text { para explicar la agresión y la } \\
\text { violencia. Unas se agrupan al } \\
\text { vincular la agresión como una } \\
\text { pulsión o fuerza interna de las } \\
\text { personas y otras al explicarla } \\
\text { desde los factores externos } \\
\text { originados en el medio ambiente. } \\
\text { Entre las teorías que se vinculan } \\
\text { con el origen de la agresión } \\
\text { desde los impulsos internos } \\
\text { del ser humano se destacan } \\
\text { la teoría de los instintos y las } \\
\text { teorías biológicas; y desde las } \\
\text { condiciones externas la teoría de } \\
\text { la frustración- agresión y la teoría } \\
\text { del aprendizaje social”. }\end{array}$ \\
\hline
\end{tabular}

Manual de convivencia
¿Qué estrategias implementaría usted para promoverla puesta en práctica de valores humanos desde las diferentes áreas?
"Ayudar en lo que necesiten"; "Conversar con los profesores sobre los tratos entre los niños que necesitan de los demás para que nadie sea grosero"; "Ayudar a los demás, compartir con los niños" "Ayudando a los compañeros cuando no entienden"; "El valor es amor que ayuda a cumplir con las demás personas, asi aprendemos a no pelear"; "Ayudar a mi abuelo a sembrar maiz"; "Actividades recreativas"; "Que no pelearan sino que se abrazan"; "Yo implementaría para ayudar a los niños y con el grupo de Buscando amor, horas de recreación para que los niños convivan en paz y armonía"; "Hablando con los alumnos dándoles consejos para que tengan una mejor actitud para que se quieran y todos vivamos en paz"; "Tolerancia"; "Valores, respeto, amor, convivencia”; "Valores en los niños, que sean solidarios, respetuosos, amigables, comprensivos con los demás niños de la escuela".
A la luz de lo expresado por los estudiantes se entiende que la solución a las situaciones que afectan la convivencia escolar entre los educandos de la ERM La Pacha es diseñar e implementar unas estrategias pedagógicas que ayuden a disminuir el número de casos de agresividad que se presentan en la misma.

Las respuestas brindadas a través de la entrevista se corresponden con los planteamientos de DíazAguado (2002) cuando afirma que para comprender los problemas existentes hoy en la convivencia escolar conviene tener en cuenta que la actual revolución tecnológica provoca una serie de cambios contradictorios y paradójicos que obligan a adaptar la escuela a esta nueva situación. En Colombia una estrategia para mejorar la convivencia escolar implementada por el gobierno nacional es la Ley de convivencia escolar como respuesta a las diferentes situaciones que se presentan en las instituciones escolares del país. 
$\cdots$

\begin{tabular}{|c|c|c|c|}
\hline $\begin{array}{l}\text { Categoría } \\
\text { de estudio }\end{array}$ & $\begin{array}{l}\text { Pregunta } \\
\text { orientadora }\end{array}$ & $\begin{array}{l}\text { Discurso de } \\
\text { los actores }\end{array}$ & $\begin{array}{c}\text { Articulación y } \\
\text { sistematización teórica }\end{array}$ \\
\hline $\begin{array}{l}\text { Política } \\
\text { educativa }\end{array}$ & $\begin{array}{l}\text { ¿Cómo cree } \\
\text { usted que } \\
\text { pueden } \\
\text { participar } \\
\text { los padres de } \\
\text { familia en la } \\
\text { realización del } \\
\text { proyecto? }\end{array}$ & $\begin{array}{l}\text { "Ayudándonos a no pelear"; } \\
\text { "Hablar con los padre de } \\
\text { familia sobre el proyecto"; } \\
\text { Diciéndoles a los padres } \\
\text { que sean amorosos con sus } \\
\text { hijos que no sean agresivos } \\
\text { con ellos"; "Que los padres } \\
\text { ayuden a los hijos cuando } \\
\text { los necesiten porque los } \\
\text { niños siempre necesitamos } \\
\text { de nuestros padres para que } \\
\text { nos brinden su amor y nos } \\
\text { guíen por el camino del bien"; } \\
\text { "Enseñándoles a no pelear } \\
\text { y a respetar a los adultos"; } \\
\text { "Cumplir los deberes con los } \\
\text { hijos"; "No tener pena con } \\
\text { nadie, ni con los estudiantes } \\
\text { ni con los profesores"; } \\
\text { "Ayudar a sus hijos a no } \\
\text { portarse mal con los demás"; } \\
\text { "Hacer lo que nos dicen en } \\
\text { la escuela"; "Invitándolo al } \\
\text { proyecto", "Que sean amables } \\
\text { que puedan jugar futbol"; } \\
\text { "Que los padres ayuden a los } \\
\text { niños si tienen problemas en } \\
\text { el colegio o en otra parte". }\end{array}$ & $\begin{array}{l}\text { Las respuestas de los estudiantes } \\
\text { se articulan con lo expresado por } \\
\text { Escamilla (2011), cuando afirma } \\
\text { que la familia es un contexto } \\
\text { de desarrollo primordial para } \\
\text { que los niños potencien toda } \\
\text { su capacidad. Éstos, aunque } \\
\text { nace bien dotados en diversas } \\
\text { áreas del desarrollo, en el } \\
\text { aspecto emocional se encuentran } \\
\text { indefensos y necesitan un lazo } \\
\text { afectivo, que en un primer } \\
\text { momento se establece con su } \\
\text { madre y distintos miembros de la } \\
\text { unidad familiar. A este vínculo se } \\
\text { le denomina apego. } \\
\text { Tal y como exponen Palacios } \\
\text { y Rodrigo (1998), "los padres } \\
\text { actúan con sus hijos, encauzan } \\
\text { su comportamiento en una } \\
\text { determinada dirección, se } \\
\text { aseguran de que no actúen de } \\
\text { una determinada manera, ponen } \\
\text { límites a sus deseos, les procuran } \\
\text { satisfacciones y les hacen soportar } \\
\text { frustraciones". Todo este grupo } \\
\text { de conductas es denominado } \\
\text { estrategias de socialización. }\end{array}$ \\
\hline Bulliyng & $\begin{array}{l}\text { ¿Con qué } \\
\text { frecuencia } \\
\text { observa } \\
\text { episodios de } \\
\text { agresividad en } \\
\text { los menores? }\end{array}$ & $\begin{array}{l}\text { "Algunas veces"; "Con } \\
\text { bastante frecuencia"; "Pegan } \\
\text { porque no obedecen a sus } \\
\text { padres, hermanos mayores } \\
\text { o familiares"; "No ver cosas } \\
\text { malas en la televisión de } \\
\text { nuestras casas y en otras } \\
\text { partes y ver cosas buenas"; } \\
\text { "Ayudando a los estudiantes } \\
\text { pequeños a hacer las tareas"; } \\
\text { "Los niños no deben buscar } \\
\text { peleas a los mayores"; "En la } \\
\text { hora de descanso, en la casa, } \\
\text { en las horas de clases"; "Antes } \\
\text { había mucha agresividad } \\
\text { cada día había una pelea por } \\
\text { eso hoy en día ya no se ve } \\
\text { tanta agresividad gracias al } \\
\text { proyecto Ciclón". }\end{array}$ & $\begin{array}{l}\text { Los estudiantes en su mayoría } \\
\text { afirman que los casos de } \\
\text { agresividad se presentan con } \\
\text { menos frecuencia que antes de } \\
\text { la aplicación y realización del } \\
\text { proyecto de investigación. } \\
\text { Reconocen que el ambiente escolar } \\
\text { ha mejorado, aunque la percepción } \\
\text { de algunos de ellos es que no ha } \\
\text { sucedido tanto así. }\end{array}$ \\
\hline
\end{tabular}

Fuente: elaboración propia. 


\section{Conclusiones}

Los hallazgos encontrados con anterioridad permiten la generación de las siguientes conclusiones:

- En cuanto a convivencia escolar, existen unos propósitos anuales en IED y es el de poder contribuir a las relaciones en armonía, la cual está siendo alcanzada gracias a las estrategias que contempla el proyecto de investigación, el compromiso y sentido de pertenencia institucional de los maestros/as.

- La aplicación de estrategias que conlleven a mejorar la convivencia escolar entre los educandos es una inversión en valores que se hace hoy para que se refleje en el futuro egresado y su comportamiento en la sociedad.

- Las familias se han comprometido e identificado con los propósitos de la propuesta y han generado estrategias a manera de sugerencias como una forma de contribuir a mejorar el ambiente escolar de la ERM La Pacha.

- Por último, se debe seguir fortaleciendo el corporativismo porque en cuanto a la relación con las familias y su participación en las actividades al interior de la institución educativa, aspecto que favorecerá las relaciones con sus hijos y promoverá la participación.

\section{Referencias}

Araque, F. y Suárez, O. (2017). Equidad Ética-Jurídica de la Ciencia, para la Emancipación del Conocimiento y los Saberes. Jurídicas CUC, 13(1). 97120. http://dx.doi.org/10.17981/juridcuc.13.1.2017.5
Avendaño, I., Cortés, O. y Guerrero, H. (2015) Competencias sociales y tecnologías de la información y la comunicación como factores asociados al desempeño en estudiantes de básica primaria con experiencia de desplazamiento forzado Diversitas: Perspectivas en Psicología, 11(1). 13-36 Obtenido de http://www.scielo.org.co/ pdf/dpp/v11n1/v11n1a02.pdf.

Carbonell, J. (2001). Escuela y entorno (Segunda ed.). Barcelona: Ediciones Morata. Obtenido de https://sites.google.com/site/portafoliodeobservac i o n $2 /$ u n i d a d - i / reporte-delectura-la-escuela-y-entorno-o-cuando-la-ciudad-tambien-edu$\begin{array}{llll}\text { c } & \text { a } & - & 1\end{array}$ reporte-de-lectura-laescuela-y-entorno-o-cuando-la-ciudad-tambien-educa

De la Concepción, A. (2015). Concepciones sobre partición de niñas, niños y adolescentes: $\mathrm{Su}$ importancia en la construcción de la convivencia escolar. Cultura Educación y Sociedad 6(2). 9-28. Recuperado de https://revistascientificas.cuc.edu.co/culturaeducacionysociedad/article/view/840

Díaz-Aguado, M. J. (2002). Cómo mejorar la convivencia escolar ante los retos de la educación del siglo XXI. Obtenido de http://www.schoolbullying.eu/doc/Convivencia_escolar. pdf

Escamilla., C. (2011). Familia y convivencia escolar. [Tesis Master]. Universidad de Almería, Almería, España. Obtenido de http://repositorio.ual.es/bitstrea m/hand-

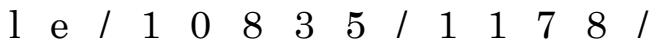
Garcia_Escamilla_Carmen_Maria. pdf? sequence $=1 \&$ isAllowed $=\mathrm{y}$ 
Grawitz, M. (2011). Métodos y técnicas de las ciencias sociales. México, D.F.: Fontana y Frey.

Guerrero, H. y Cera, J.(2015). Impacto laboral y social de los especialistas en estudios pedagógicos-CUC. Opción, 31 (No. Especial 6). 1160-1174..Recuperado de http://www.produccioncientifica. luz.edu.ve/index.php/opcion/article/ viewFile/20781/20661

Guerrero. H, y Cepeda, M. (2016) Uso de estrategias pedagógicas para el fortalecimiento de la convivencia escolar de jóvenes vulnerables. Revista de Pedagogía, 37(101). 57-79. Recuperado de http:// saber.ucv.ve/ojs/index.php/rev_ped/article/view/12501.

Guerrero. H, y Cepeda,M.(2016). El impacto del lider comunitario en el siglo XXI. Barranquilla: Editorial Universitaria de la Costa, Educosta.
Hernández, C. (2006). Metodología de la investigación (4 ed.). México, D.F.: McGraw-Hill. Obtenido de http://redes.colombiaaprende.edu.co/ntg/men/pdf/ Guia\%20No.\%2049.pdf http://trabajosocialmazatlan.com/multimedia/files/InvestigacionPosgrado/Entrevista.pdf

Jiménez, E. (2008). El papel del profesorado en la actualidad. Su función docente y social. Foro de Educación, 10. 325345. Recuperado de https://dialnet.unirioja.es/descarga/articulo/2907073.pdf

Martìnez, J., Berthel, Y. y Vergara, M. (2017). Síndrome de Burnout en profesores y su relación con el aprendizaje de los estudiantes de básica primaria de una institución educativa oficial de Sincelejo (Colombia), Salud Uninorte. Barranquilla (Col.) 33(2): 118-128. Recuperado de http://www.scielo.org.co/ $\mathrm{pd} \mathrm{f/s} \mathrm{u} \mathrm{n} \mathrm{/} \mathrm{v} 33$ n $2 / 2011$ - 7531 sun-33-02-00118.pdf 